\title{
Zur transnationalen sozialen Frage: Soziale Ungleichheiten durch soziale Sicherung in Europa
}

\author{
Grenzübergreifende soziale Sicherung und Mobilität
}

In Europa lässt sich ein wachsender, zumindest aber anhaltend hoher Grad sozialer Ungleichheit feststellen. Diese Ungleichheit betrifft zum einen die wachsende Kluft zwischen den Einkommen, zwischen Lohneinkommen und Gewinneinkommen, Einkommen aus Arbeit und Einkommen aus Kapitalanlagen, aber zum anderen auch zwischen den Lebenschancen in den verschiedenen Regionen Europas. Inwiefern, so die Frage im folgenden Beitrag, können öffentliche beziehungsweise private Strategien sozialer Sicherung und grenzübergreifender Mobilität die ungleich verteilten Lebenschancen beeinflussen? ${ }^{2}$

Dazu wird auf zwei Beispiele transnationaler Migration zurückgegriffen, die unterschiedliche Dimensionen betreffen. Ein Beispiel betrifft Pflegekräfte aus der Ukraine und aus Rumänien, die in italienischen oder deutschen Haushalten arbeiten, um Einkommen für sich und ihre Familien zu erwerben. Teilweise pendeln sie dabei zwischen Zielort und Herkunftsort und sind in vielfältige grenzübergreifende Transaktionen eingebunden, so etwa im Hinblick auf Überweisungen für Schulgebühren, Krankenversorgung oder Immobilien, Kinderbetreuung aus der Nähe und der Ferne und Pflege von Familienangehörigen beziehungsweise von Personen im Haushalt des Immigrationslands. Gleichzeitig tragen sie zur Reproduktion der Arbeitskraft der Haushalte bei, in denen sie arbeiten, beispielsweise durch die Betreuung von Kindern und durch die Pflege älterer Angehöriger in den Zielregionen, indem sie die Familien entlasten und den arbeitsfähigen Familienangehörigen, meist den Frauen, erlauben, ihre Arbeitskraft zu Markte zu tragen.

Das andere Beispiel betrifft einen stark männlich geprägten Sektor, die Bauwirtschaft, in dem Werkvertragsarbeitnehmer oder Selbstständige aus EU-Mitgliedstaaten oder aus Drittstaaten Dienstleistungen erbringen. Hier stellt sich etwa bei Selbstständigen die Frage nach sozialer Sicherung im Falle von Arbeitsunfällen, bei Werkvertragsarbeitnehmern die Frage nach Bezahlung und Arbeitsbedingungen im Vergleich zu einheimischen Arbeitern und in beiden Fällen die Frage nach sozialer Sicherung der im Herkunftsland verbliebenen Angehörigen.

1 Der Autor dankt Karolina Barglowski, Başak Bilecen, Eveline Reisenauer, Joanna Jadwiga Sienkiewicz, Christian Ulbricht und den Gutachtern dieser Zeitschrift für hilfreiche Kritik. Dank gebührt Reinhard Blomert für zahlreiche Verbesserungsvorschläge.

2 Siehe unter anderem Heidenreich 2006; Berger, Weiß 2008; Beck, Proferl 2010. 
Arbeit wird teilweise sowohl im Zielland als auch im Herkunftsland erbracht, und soziale Sicherung wird je nachdem nicht nur innerhalb des Gebiets eines einzelnen nationalen (Wohlfahrts-)Staates bereitgestellt beziehungsweise nachgefragt und in Anspruch genommen. Häufig sind Arbeitsverhältnisse in den genannten Sektoren irregulär, und in den Herkunftsregionen ist beispielsweise die Betreuung von Familienangehörigen wie Kindern oder Älteren nicht gesichert - dann stellen sich Fragen nach Ungleichheiten in den betroffenen Haushalten in den Ziel- und Herkunftsregionen, etwa beim Zugang zu sozialen Rechten, bei der Möglichkeit der Nutzung von Bankkonten für die Überweisungen aus den und in die Herkunftsregionen oder bei der Kindererziehung und in den Machtbeziehungen zwischen den Generationen in den Haushalten.

Die folgende Analyse arbeitet soziale Mechanismen heraus, die im grenzübergreifenden, das heißt transnationalen Kontext in Europa - sowohl der Europäischen Union (EU) als auch der Anrainerstaaten - ungleichheitsrelevant wirken. Transnational ist hier ein Oberbegriff für grenzübergreifende Interaktionen, die drei mögliche Ebenen mit einschließen: zwischenstaatliche Beziehungen (international), Beziehungen in einem Staatenverbund (supranational) oder weltweit (global). Dabei bezieht sich der Begriff »transnational « immer auch auf nichtstaatliche Akteure und betrifft Prozesse der Vergemeinschaftung und Vergesellschaftung im transnationalen Rahmen, die ich » Transnationalisierung « nenne. Die durch Prozesse der Transnationalisierung entstandenen grenzübergreifenden sozialen Verdichtungsräume werden hier » transnationale soziale Räume « genannt. Die »Transnationalität « wird als messbar begriffen und als Ausmaß an grenzübergreifenden Transaktionen von Personen und Gruppen entlang eines Kontinuums von niedrig bis hoch verstanden. ${ }^{3}$

Transnationalität, so die Ausgangsvermutung, ist sowohl mit der Abmilderung bestehender als auch der Generierung neuer Ungleichheiten verbunden. Der Fokus liegt hier darauf, in welchem Verhältnis grenzübergreifende soziale Sicherung zu den innereuropäischen sozialen Ungleichheiten steht; es geht also um die Verteilung wertvoller Ressourcen, um Status und Macht, die für Lebenschancen entscheidend sind. Dies erfordert einen Blick sowohl auf nationale und europäische Politik als auch die Praktiken von Familien und Netzwerken in den transnationalen sozialen Räumen.

Grenzübergreifende Mobilität ist ein Forschungsbereich, in dem die Problematik der sozialen Absicherung in nationalen Wohlfahrtsstaaten untersucht wird. Denn die Beschäftigung von Personen, die grenzüberschreitend mobil sind, hat Auswirkungen auf ihre soziale Sicherung in mehr als nur einem Staat und wirkt sich auf verschiedene soziale Gruppen aus. Migranten ${ }^{4}$ und andere mobile Arbeitskräfte, etwa die an zirkulärer Mobilität beteiligten Saisonarbeiter, Werkvertragsarbeitnehmer oder auch Manager in multinationalen Unternehmen, unterhalten und pflegen

3 Faist et al. 2013, Kapitel 1.

4 Aus Gründen der Lesbarkeit wird auf die Nennung der weiblichen Form verzichtet. Im Folgenden impliziert die männliche Form jedoch stets die gleiche Berücksichtigung der weiblichen Form. 
häufig enge und dauerhafte Bindungen an ihre Herkunftsorte, die auf soziale Bindungen an Personen, Gruppen und Organisationen zurückgehen. Die Lebenswelten dieser Beschäftigten sind also über die Grenzen von Staaten hinweg organisiert. Dies lässt sich auch statistisch erfassen: Eine Analyse von Daten des Sozio-oekonomischen Panels (SOEP) ergibt beispielsweise für das Jahr 2010, dass 80 Prozent aller in Deutschland ansässigen Migranten grenzübergreifende Transaktionen in verschiedenen Formen vornehmen, etwa Geld überweisen, Reisen unternehmen sowie Kontakte zu Freunden und Verwandten im Ausland unterhalten. Bei Nicht-Migranten, das heißt deutschen Staatsbürgern ohne Migrationshintergrund, sind es im Vergleich nur etwas über 30 Prozent. ${ }^{5}$

Die grundlegende These ist, dass Arbeitsmigration und Formen grenzübergreifender sozialer Sicherung eine Antwort auf soziale Risiken und damit zusammenhängende Ungleichheiten der Chancen sind, sie aber zugleich alte Ungleichheiten reproduzieren und neue schaffen. Dies ist auch im Zusammenhang damit zu sehen, dass grenzübergreifende Migration, soweit sie nicht erzwungen ist, ein Resultat sozial-ökonomischer, kultureller und politischer Transformation und den damit zusammenhängenden, zum Teil sogar wachsenden Ungleichheiten zwischen Weltregionen ist. Migration trägt daher gerade nicht, wenigstens nicht direkt, zum Abbau von regionalen Ungleichheiten bei. ${ }^{6}$ Dem widerspricht nicht, dass auf individueller und familiärer Haushaltsebene geografische Mobilität und damit verbundene Transnationalität eine durchaus erfolgreiche Strategie zur Erlangung von Beschäftigung und sozialer Sicherung sein mag. ${ }^{7}$ Nichtsdestotrotz werden, wie noch zu zeigen sein wird, auch auf dieser Ebene durchaus Ungleichheiten (re)produziert.

Die transnationale soziale Frage betrifft also Prozesse der Wahrnehmung und Politisierung von sozialen Ungleichheiten zwischen Staaten vor dem Hintergrund von Forderungen nach mehr sozialer Gleichheit in grenzübergreifender Hinsicht, sowohl regional als auch weltweit. Die transnationale soziale Frage in Europa ist in gewisser Weise eine regionale Ausprägung der globalen sozialen Frage, mit dem Unterschied, dass Europa sich als eine Union von zivilisierten Wohlfahrtststaaten definiert (auch wenn dieses Charakteristikum keineswegs auf alle Mitgliedstaaten in gleicher Weise anwendbar ist). Dieser Unterschied bildet jedoch den Hintergrund dafür, dass soziale Ungleichheiten in öffentlichen Debatten in Europa auf der Grundlage von sozialen Menschenrechten - kodifiziert etwa in der Europäischen Konvention zum Schutz der Menschenrechte und Grundfreiheiten (Rom 1950), der Europäischen Sozialcharta des Europarats (Turin 1961) und der Europäischen Sozialrechtscharta (1989) - auch grenzübergreifend als ausgleichswürdig wahrgenommen werden. Deshalb können wir also von einer transnationalen sozialen Frage in Europa sprechen. So fordern etwa globalisierungskritische soziale Bewegungen eine verstärkte Bekämpfung von Armut und Zugang zu Gesundheits- und Bildungsein-

5 Fauser et al. 2012. Siehe dazu auch weitere Ergebnisse aus dem Teilprojekt »Transnationalität und Ungleichheiten: Pilotprojekt Panelstudie « im SFB 882 »Von Heterogenitäten zu Ungleichheiten « (www.sfb882.uni-bielefeld.de/de/projects/c1).

6 Castles, Miller 2009.

7 Goldin et al. 2012. 
richtungen für alle. ${ }^{8}$ Derartige Forderungen beruhen auf der Annahme einer unaufhaltbaren, ja sogar wünschbaren wachsenden Interdependenz zwischen verschiedenen Regionen in Europa und der normativen Bewertung der Illegitimität starker sozialer Ungleichheiten. ${ }^{9}$ Die Foren, in denen Wahrnehmungen sozialer Ungleichheit und die Forderungen nach sozialer Gleichheit über Staatsgrenzen hinweg artikuliert werden, sind freilich ebenso vielfältig wie diejenigen, die sich daran beteiligen. Dazu zählen Stimmen im Rahmen der Öffentlichkeit in den einzelnen Nationalstaaten, aber auch Stimmen innerhalb der supranationalen Institutionen wie der EU, in internationalen Organisationen wie der Internationalen Arbeitsorganisation (ILO) und, nicht zuletzt, in sozialen Bewegungsorganisationen und Netzwerken, die im (regional organisierten) Weltsozialforum tätig sind.

Der erste Teil dieses Beitrags umreißt die transnationale soziale Frage in Europa als Problem der Ungleichheit auf der Folie geltender oder gewünschter Gleichheitsnormen im Rahmen nationaler sozialer Bürgerschaft und in der Europäischen Union. Der nationale und der supranationale Rahmen sind jedoch nicht hinreichend, um die Folgen beziehungsweise Möglichkeiten sozialer Sicherungsstrategien zu erklären. Daher ist im zweiten Teil ein transnationaler Blick notwendig, mit dem die Relevanz von grenzübergreifenden sozialen Beziehungen von Migranten und Nicht-Migranten in transnationalen sozialen Räumen für Lebenschancen und Ungleichheiten gezeigt werden kann. Im dritten Teil werden soziale Mechanismen betrachtet, die soziale Ungleichheiten nicht nur nicht abbauen, sondern im Gegenteil noch verstärken beziehungsweise neue Ungleichheiten generieren. Dafür werden Beispiele sowohl aus dem Haushalts- und Pflegebereich als auch aus der Bauwirtschaft herangezogen. Schließlich werden die aus der empirischen Analyse sich ergebenden Forschungsfragen aufgeführt.

\section{Gleichheitsnormen und soziale Bürgerschaft}

Ungleichheiten im Europa der Gegenwart erinnern an die »soziale Frage ", die im 19. Jahrhundert in den Industrieländern auf dem Kontinent das zentrale Thema der politischen Konflikte zwischen den herrschenden Klassen und den sozialen Bewegungen der Arbeiterklasse war. ${ }^{10}$ Angesichts der Ungleichgewichte in den verschiedenen Staaten Europas im Hinblick auf Einkommen im Speziellen und Lebenschancen im Allgemeinen könnte man fragen, ob wir nun am Rande eines neuen sozialen Konflikts stehen, der diesmal auf transnationaler Ebene und entlang vielerlei Grenzziehungen ausgetragen wird - eben nicht nur, wie es für das Europa des 19. Jahrhunderts galt, entlang von Klassengrenzen, also in den Kategorien Kapital und Arbeit, sondern vermehrt auch entlang von Differenzen beziehungsweise Heterogenitätsmerkmalen wie Geschlecht, Rasse, Ethnizität, Rechtsstatus, geschlechtliche Orientierung, Religion und nicht zuletzt Transnationalität. Einen Hinweis auf damit

8 Cabrera 2001; Fischer-Lescano, Möller 2012.

9 Heidenreich, Wunder 2008.

10 Siehe De Swaan 1988.

Leviathan, 41. Jg., 4/2013 
verbundene Konflikte geben die Forderungen politischer Gruppierungen und Nichtregierungsorganisationen (NROs), die über nationale Grenzen hinweg in verschiedenen Kampagnen für Themen wie Umwelt, Menschenrechte oder Genderfragen aktiv sind. ${ }^{11}$

Es kann nun nicht vorausgesetzt werden, dass die Betroffenen ebenso wie die politischen und wirtschaftlichen Entscheidungsträger und die Aktivisten die gleiche Wahrnehmung in Bezug auf die Bewältigung von Ungleichheiten haben. Auch, dass es eine Art natürlicher Evolution globaler sozialer Normen gibt, die etwa durch supranationale soziale Rechte und soziale Bürgerschaft über nationale Grenzen hinweg durchsetzbar wären, kann man nicht einfach annehmen. So sind beispielsweise grenzübergreifende Politikmaßnahmen, die gravierende Ungleichheiten auszugleichen bestrebt sind und dabei Aspekte sozialer Sicherung behandeln, über den einzelnen Nationalstaat hinaus auf europäischer Ebene häufig nicht in klar einklagbare soziale Rechte eingegossen. Auf supranational-europäischer Ebene existieren nur wenige kodifizierte soziale Rechte, sieht man einmal von Bestimmungen etwa zu geschlechtlicher Gleichberechtigung (Artikel 141 EG-A), Gesundheit und Sicherheit am Arbeitsplatz und Migration von Bürgern der Mitgliedstaaten innerhalb der EU ab. ${ }^{12}$ Häufig handelt es sich bei grenzübergreifenden sozialen Rechten um nicht einklagbare Normen (soft law), also etwa soziale Standards, die Arbeitsbedingungen auf freiwilliger Basis in multinationalen Konzernen regeln, oder um Politikmaßnahmen, die indirekt Einkommen generieren und soziale Sicherung betreiben, zum Beispiel die Gemeinsame Agrarpolitik der EU.

Die »neue « soziale Frage kann dabei als ein Prozess der Herausbildung von Diskursen und Politikmaßnahmen verstanden werden, in denen es in öffentlichen Debatten auf nationaler Ebene (zum Beispiel in sozialen Bewegungen, Wohlfahrtsverbänden und in staatlichen Einrichtungen), auf supranationaler Ebene (etwa im Europäischen Parlament und in der EU-Kommission) und auch in globalen Kontexten (beispielsweise UN, Weltsozialforum) um die Wahrnehmung und Bekämpfung von grenzübergreifend relevanten Ungleichheiten auf der Folie von Normen der Gleichheit geht, wie sie prominent in der Erklärung für Menschenrechte und

11 Carver, Bartelson 2011.

12 Faist 2000. 
verschiedenen UN-Konventionen ihren Ausdruck gefunden haben. ${ }^{13}$ Von einer transnationalen sozialen Frage kann gesprochen werden, wenn grenzübergreifende Ungleichheiten vor dem Hintergrund der Forderungen nach Gleichheit infrage gestellt werden. Voraussetzung dafür ist die öffentlichkeitsrelevante Wahrnehmung von Interdependenzen, die quer zu nationalstaatlichen Grenzen liegen. In einer grenzübergreifenden Öffentlichkeit ist etwa an die Proteste von sozialen Bewegungen im Rahmen des Weltsozialforums zu denken und auf europäischer Ebene beispielsweise an die Aktivitäten von NROs im Rahmen der EU (zum Beispiel Inequality Watch). Ungleichheiten werden, so eine grundlegende Einsicht der soziologischen Ungleichheitsforschung, erst vor dem Hintergrund von Normen der Gleichheit als Ungleichheiten wahrgenommen. ${ }^{14}$ Im nationalen Rahmen sind Normen der Gleichheit in den Verfassungen enthalten, darüber hinaus in der Charta der Grundrechte der EU und der Allgemeinen Erklärung der Menschenrechte der Vereinten Nationen, die einen globalen Anspruch haben. Allgemein akzeptierte Normen der Gleichheit, die sich auf gleiche soziale Sicherung auch bei grenzübergreifenden Arbeitsverhältnissen beziehen könnten, gibt es im Sinne kodifizierter und rechtlich einklagbarer Normen allerdings nicht. Somit ist neben menschenrechtlichen Normen für die Betrachtung des Zusammenhangs von Ungleichheiten und Normen der Gleichheit zunächst einmal die inzwischen klassische Diskussion über die soziale Bürgerschaft (social citizenship) ein sinnvoller Ausgangspunkt. T. H. Marshall sah national institutionalisierte soziale Bürgerschaft als möglichen Weg, um mittels sozialer Sicherung die von Marktteilhabe und Marktausgrenzung ausgehenden Risiken zu mindern und damit (fort)bestehende soziale Ungleichheiten zu legitimieren. Soziale Bürgerschaft galt für ihn als ein egalitärer Status, die mit der vollen Mitgliedschaft zu einer nationalen Gesellschaft verbunden war. Nach Marshall stärken soziale Rechte und Sozialpolitik den wohlfahrtsstaatlichen Kapitalismus auf nationaler Ebene und grenzen Verteilungskonflikte ein. ${ }^{15}$

Durch grenzübergreifende Mobilität von Menschen, den Tausch von Waren, die Diffusion von Ideen und die Erbringung von Dienstleistungen wird diese nationale wohlfahrtsstaatliche Konstellation, die soziale Rechte garantiert und damit auch

13 Beispiele dafür sind die Allgemeine Erklärung der Menschenrechte (1948) und der Internationale Pakt über wirtschaftliche, soziale und kulturelle Rechte (1966). Die wichtigsten sozialen Rechte (Artikel 22-27) in der Allgemeinen Erklärung der Menschenrechte sind ein grundlegendes Recht auf Schulbildung, das Recht auf Arbeit und das Recht, einer Gewerkschaft beizutreten, das Recht auf ein minimales Einkommen, Nahrung, Kleidung, Wohnung, medizinische Versorgung und soziale Absicherung. Auf Migranten bezogen, fanden diese Rechte beispielsweise in der Konvention zu Wanderarbeitnehmern der Internationalen Arbeitsorganisation (ILO) von 1949 oder der Internationalen Konvention zum Schutz der Rechte aller Wanderarbeitnehmer und ihrer Familienangehörigen der Vereinten Nationen (UN) von 1990, die neben den Rechten von Arbeitern auch die ihrer Familien mit einschließt, Ausdruck. Zu einem Überblick zu sozialer Sicherung und Migration aus institutioneller Sicht siehe unter anderem Crevits, Van Buggenhout 2005, S. 33-41.

14 Hondrich 1984.

15 Marshall 1964 [1950].

Leviathan, 41. Jg., 4/2013 
einen Horizont für die Wahrnehmung und Politisierung von Ungleichheiten bildet, in zweierlei Hinsicht aufgebrochen: zum einen durch neue Probleme für die soziale Sicherung von Nicht-Bürgern, von denen zwar etliche sesshaft sind, aber häufig nicht über volle soziale Rechte verfügen. ${ }^{16}$ Innerhalb der EU verfügen sesshafte Nicht-Bürger aus Drittstaaten (Wohnbürger) zumeist nicht über die gleichen sozialen Rechte wie mobile Bürger aus den Mitgliedstaaten. Zum anderen gilt es, die zu Nationalstaaten und supranationalen Strukturen quer liegenden transnationalen sozialen Räume und damit die Transnationalität der Praktiken mit zu berücksichtigen, um die tatsächlichen Konsequenzen sozialer Sicherung für Ungleichheiten zu erfassen.

Suprastaatliche Entwicklungen in der EU geben erstens einen Einblick in das komplexer werdende Zusammenspiel mehrerer Ebenen der Regulierung sozialer Rechte von Migranten und grenzüberschreitend mobilen Beschäftigten. Die Kopplung verschiedener nationaler Systeme auf europäischer Ebene birgt nun unterschiedliche Lebenschancen für Bürger von Mitgliedstaaten und Drittstaaten, selbst wenn letztere in einem EU-Staat einen dauerhaften Wohn- und Arbeitsmarktstatus innehaben. Denn ist der Grad an Institutionalisierung sozialer Rechte auf EU-Ebene schon gering, so wird es nicht verwundern, dass es zudem große Unterschiede zwischen grenzüberschreitend mobilen EU-Bürgern (beispielsweise französische Bürger, die in Italien arbeiten) und Nicht-EU Bürgern (etwa ukrainische Bürger in Deutschland) gibt: Während EU-Bürger als Arbeitnehmer von Anfang an weitgehend gleiche soziale Rechte in einem anderen Mitgliedsland in Anspruch nehmen können, trifft das auf Drittstaatenangehörige nicht zu. Für diese gilt, dass sie derartige Ansprüche erst im Rahmen eines gesicherten Aufenthalts- und Arbeitsstatus erlangen können und somit etwas erreichen, was als Wohnbürgerstatus bezeichnet werden kann. An dieser Stelle wird schon deutlich, dass im Hinblick auf soziale Rechte auf EU-Ebene eine Koordination nationaler Rechtsansprüche für EU-Bürger zu beobachten ist (zum Beispiel in Form der Übertragbarkeit sozialer Ansprüche über Staatsgrenzen hinweg), während für die extracommunitari der Status der jeweiligen binationalen Abkommen gilt. ${ }^{17}$ Eine Harmonisierung im Sinne einer Angleichung des Status von Drittstaatenangehörigen an EU-Bürger ist nicht in Sicht. Der Grund dafür liegt darin, dass nationale Wohlfahrtsstaaten die Kontrolle über ihre Arbeitsmärkte und ihre Systeme der sozialen Sicherung nicht an supranationale Institutionen abgeben wollen, zumal aus EU-Sicht die Konkurrenz zwischen den Mitgliedstaaten mit der Folge geringerer sozialer Rechte und höherer internationaler Wettbewerbsfähigkeit wünschenswert ist. Dies lässt sich leicht am Beispiel der Freizügigkeit der Arbeitskräfte zeigen: Argentinier mit italienischer Abstammung können die Staatsangehörigkeit ihrer Vorfahren annehmen; sie verfügen aber damit über die Möglichkeit, sich nicht nur in Italien niederzulassen, sondern auch in jedem anderen EU-Mitgliedsland. Oder betrachten wir Personen aus Moldawien, welche

16 Morris 2002.

17 Assoziationsabkommen mit einzelnen Anrainerstaaten, insbesondere der Türkei, verweisen auf eine etwas privilegiertere Position der Bürger aus diesen Staaten; siehe Groenendijk et al. 2013. 
die rumänische Staatsangehörigkeit erhalten können, was ihnen Freizügigkeit in der EU sichert. In diesen und ähnlich gelagerten Fällen können die übrigen Mitgliedstaaten der EU keine Kontrolle über die Mobilität von Arbeitskräften qua Staatsangehörigkeit ausüben. Allerdings haben sie diese Kontrollmöglichkeit bei den Drittstaatenangehörigen. Mitgliedstaaten nutzen also Migrationskontroll- und teilweise Einbürgerungspolitik im Hinblick auf Drittstaatenangehörige dazu, um ihre Arbeitsmärkte (und damit den Preis der Arbeitskraft) und die soziale Bürgerschaft zu regulieren. Denn dieses Souveränitätsrecht steht ihnen zu, während die Definition dessen, was als »Arbeitnehmer " gilt, immer mehr von der EU-Kommission selbst geschaffen und geprägt wird. Zugangspolitik wird zu einem indirekten Instrument der Kontrolle von Arbeitsmärkten und sozialen Rechten.

Die EU stellt hier ein singuläres Regime dar. Grenzübergreifende Mobilität von Beschäftigten kann als internationale, also zwischenstaatliche Migration angesehen werden, obwohl der europarechtliche Rahmen inzwischen dem von staateninterner Migration entspricht. Dieses Charakteristikum wird dadurch betont, dass EU-Institutionen wie Kommission und Parlament grenzüberschreitende Bewegungen von EU-Bürgern als »Mobilität « bezeichnen, während mit »Migration « in der Regel die grenzüberschreitenden Bewegungen von Drittstaatenangehörigen gemeint sind. ${ }^{18}$ "Mobilität « ist somit gewissermaßen Migration Erster Klasse, ohne zeitraubende Kontrollen, Visa und Aufenthaltsbeschränkungen, die kennzeichnend für internationale Mobilität beziehungsweise Migration zwischen der EU und Drittstaaten sind.

Zweitens sind all diese auf nationale Wohlfahrtstaaten, auf die institutionelle Architektur der EU und internationale Konventionen bezogenen Überlegungen zweifelsohne notwendig für das Verständnis der rechtlich-politischen Aspekte sozialer Bürgerschaft und des Zugangs zu sozialen Rechten. Sie sind jedoch nicht hinreichend, um die sozialen Ungleichheiten als grenzübergreifende Phänomene zu erfassen. Die Begrenzung auf eine nationale und supranationale Perspektive nimmt lediglich die Immigrationsseite in den Blick und blendet die lateralen Bindungen über Staatsgrenzen hinweg aus. Um diese in den Blick zu bekommen, bedarf es einer transnationalen Sichtweise jenseits von Instrumenten wie bilateralen Verträgen, es muss die Ebene der (informellen privaten) sozialen Sicherungsmechanismen zwischen Migranten und deren Angehörigen in transnationalen sozialen Räumen mit einbezogen werden. ${ }^{19}$ Daraus ergibt sich auch, dass zum Verständnis von sozialer Sicherung im Rahmen von grenzübergreifenden Transaktionen ein Fokus darauf gelegt werden muss, wie soziale Sicherung tatsächlich bereitgestellt wird und welche Folgen für soziale Ungleichheiten dabei entstehen.

Ein transnationaler Ansatz steht einem methodologischen Nationalismus, das heißt der in den Sozialwissenschaften oftmals nicht explizit gemachten Annahme, dass nationale Gesellschaften oder Nationalstaaten die natürliche Untersuchungseinheit für Analyse und Datensammlung seien, kritisch gegenüber. Über diese wohlfeile Kritik hinaus müssen jedoch die Referenzpunkte für transnationale Analysen

18 Faist, Ulbricht 2014.

19 Vgl. Sabates-Wheeler, Feldman 2011.

Leviathan, 41. Jg., 4/2013 
explizit benannt werden. ${ }^{20}$ Ein transnationaler Ansatz geht einerseits vom Grad beziehungsweise der Dichte der grenzübergreifenden Transaktionen aus, also der Transnationalität. Andererseits geht es im Rahmen dieses Ansatzes darum, jeweils verschiedene Analyseebenen wie die lokale, die nationalstaatliche, die europäische und die globale in den Blick zu nehmen - und möglichst auch deren Wechselbeziehungen. Alle diese Ebenen können Anknüpfungspunkte für grenzübergreifende Transaktionen sein.

Bei grenzübergreifenden Interaktionen sollte der Aspekt der räumlichen Mobilität der grenzüberschreitend Beschäftigten nicht als alleiniges Merkmal hervorgehoben werden. Die Rolle relativ immobiler Haushalts- oder Verwandtschaftsangehöriger, die zu Hause bleiben und die grenzübergreifende Mobilität beziehungsweise Migration anderer Mitglieder der Familie häufig erst ermöglichen, müssen auch ausreichend in Betracht gezogen werden, wenn man das Phänomen erfassen will. Nicht alle Individuen und Gruppen, die grenzübergreifende Praktiken pflegen, überqueren (regelmäßig) die Grenzen von zwei oder mehr Nationalstaaten. Es sind also nicht nur die jeweils um- und angesiedelten Migranten, sondern auch deren mitunter immobile, zu Hause gebliebene Familienmitglieder, ebenso wie schon länger niedergelassene Mitglieder einer Migrantengruppe, die grenzübergreifend aktiv sind..$^{21}$ Damit wird der Nutzen des Konzepts »Transnationalität « deutlich. Transnationalität bezeichnet die sozialen Aktivitäten von Menschen und Menschengruppen über nationalstaatliche Grenzen hinweg. Der Begriff beschreibt ein Spektrum grenzübergreifender Transaktionen in verschiedenen Sphären des sozialen Lebens familiären, soziokulturellen, wirtschaftlichen und politischen -, welches von Reisen über das Versenden von Geld (»Remissionen«) und den mit dieser Praxis verbundenen multiplen Bindungen bis hin zum Gedanken- und Ideenaustausch reicht. So gesehen, weisen transnationale Bindungen Kennzeichen der sozialen Heterogenität auf, ähnlich anderer Kennzeichen wie Alter, Geschlecht, Staatsbürgerschaft, sexuelle Orientierung, kulturelle Vorlieben, Sprache und Sprachgebrauch. Transnationalität kann also verschiedene Formen annehmen und ein Kontinuum von schwacher bis starker Betroffenheit aufweisen, das heißt von sehr wenigen und kurzlebigen bis hin zu vielfältigen, dichten und lang andauernden Bindungen. So lässt sich etwa an den Remissionen ablesen, wie stark die Bindungen an die Heimat sind. Migranten überweisen eben unterschiedlich hohe Geldbeträge - manche viel, manche vielleicht überhaupt kein Geld - an ihre Familien in der Heimat. Für unsere Zwecke heißt das auch, dass man grenzüberschreitend beschäftigte Bevölkerungen, also die nichtmigrierten Angehörigen und ihr Umfeld, nicht einfach als transnational oder nichttransnational kategorisieren kann, sondern dass Transnationalität in unterschiedlichem Maße entlang eines gedachten Kontinuums auftritt.

Die sozialen Sicherungsgefüge enthalten formelle und informelle, staatliche und nichtstaatliche Dimensionen. Im kleineren Maßstab gibt es nichtformalisierte pri-

\section{Faist 2012.}

21 Darüber hinaus sind auch bei Menschen, die nicht mit grenzübergreifender Migration in Verbindung stehen, durchaus transnationale Praktiken beobachtbar; siehe dazu Mau 2007. 
vate Freundschaftsnetzwerke ebenso wie hoch institutionalisierte verwandtschaftliche beziehungsweise familiäre Gruppennetzwerke. Unter Verwandten kann die Entscheidung zur Abwanderung als eine Art Versicherung gegen Verarmung im Sinne einer Reziprozität (also gegenseitiger Hilfestellungen im Hinblick auf vielfältige soziale Risiken) betrachtet werden, um beispielsweise für zusätzliches Einkommen zu sorgen, falls etwa unerwartete Ausgaben für Krankheiten anfallen. Gerade informellen privaten Formen von sozialer Sicherung kommt große Bedeutung in einem Zustand zu, in dem die formalen sozialen Sicherungen nicht vorhanden sind oder versagen. Dazu gehören eben gerade die Remissionen, die Transfers innerhalb der Verwandtschaft, welche beispielsweise Immobilienerwerb, Altersvorsorge oder das Studium eines Verwandten ermöglichen. Kinderbetreuung wird in Einwanderungsländern ja keineswegs immer durch kommunale Kindertagesstätten gewährleistet, sondern muss teilweise durch Verwandte geleistet werden, die aus dem Ausland anreisen und sich um die Kinder kümmern, sodass deren Mütter und Väter außerhalb des Hauses arbeiten können. Manchmal werden auch ältere Verwandte, die im Heimatland nicht versorgt oder gepflegt werden können, nachgeholt.

Die hier privilegierte Perspektive befasst sich mit Ungleichheiten im Kontext der grenzübergreifenden Transaktionen von Individuen und Gruppen. Die Beobachtungs- und Analyseeinheiten hängen von der jeweiligen Fragestellung ab, weil Transaktionen auf verschiedenen Ebenen stattfinden können, etwa in Haushalten, lokalen oder religiösen Gemeinschaften oder Organisationen.

\section{Ungleichheitsrelevante Mechanismen}

Die Praxis der Entstehung von Ungleichheiten über die Grenzen des Nationalstaats hinaus werden am Beispiel der Kommodifizierung von Arbeitskraft sichtbar: In diesem Prozess werden aus naturwüchsigen Beziehungen der Arbeitsteilung monetarisierte Marktbeziehungen. Wie bereits ausgeführt, unterliegen verschiedene Sphären der Arbeit wie etwa die Langzeitpflege älterer Menschen und Hausarbeit in einigen EU-Mitgliedstaaten und anderen OECD-Ländern einer wachsenden Kommodifizierung. Bei der Pflege- und Hausarbeit handelt es sich um eine vielschichtige Arbeit, welche die täglichen Lebensbedingungen reproduziert, die Gesundheit und Wohlfahrt überhaupt erst ermöglichen. Haus- und Pflegearbeit, sowohl die naturwüchsige als auch die kommerzielle, ist derzeit hauptsächlich ein Feld für weibliche grenzüberschreitend Beschäftigte. Das geht unter anderem darauf zurück, dass in der OECD der Anteil der Erwerbstätigkeit von Frauen in formalen Arbeitsverhältnissen steigt. Eine Folge davon ist, dass diese für die Pflege- und Hausarbeiten in den Ländern des Zentrums nicht mehr in ausreichendem Umfang zur Verfügung stehen. ${ }^{22}$ Pflege- und Betreuungsarbeit hat sich daher inzwischen zu einem expandierenden Beschäftigungsfeld entwickelt - laut ILO sind weltweit über 100 Millionen Migranten in diesem Bereich beschäftigt -, und der Anteil älterer Menschen,

22 Pfau-Effinger 2000.

Leviathan, 41. Jg., 4/2013 
die solcher Pflege bedürfen, nimmt zu, je mehr Frauen sich für den Weg in den industriellen, Handels- oder Büroarbeitsmarkt entscheiden.

Als weiteres Beispiel einer Kommodifizierung kann der Bausektor gelten. Der Anteil von (abhängigen) Selbstständigen in westeuropäischen Staaten im Baugewerbe ist gerade seit dem Beitritt mittel- und osteuropäischer Länder zur EU und der damit verbundenen Freizügigkeit für Arbeitnehmer in den letzten Jahren stark angestiegen. Dabei ist ein großer Teil dieser Selbstständigen nicht oder nur ungenügend sozialversichert. Konkret werden bei den auftretenden Formen von abhängiger Selbstständigkeit zumeist unter anderem Regeln des Kündigungsschutzes und die Aufhebung der Befristung von Verträgen umgangen. Häufig ist das Arbeitsrecht dann nicht anwendbar, weil privatrechtliche Verträge abgeschlossen wurden, was bedeutet, dass faktisch weisungsabhängige Arbeitnehmer vertraglich wie tarifunabhängige Selbstständige behandelt werden. ${ }^{23}$ Eine langfristige Konsequenz ist eine nicht hinreichende Vorsorge für das Alter.

Strategien grenzübergreifender Mobilität verbinden soziale Sicherung in den Ursprungsländern mit dem Zielland. Entsprechende relevante Regulierungen in der Sozial- und in der Migrationspolitik der Länder bilden jedoch kein in sich schlüssiges grenzüberschreitendes soziales Integrationsprogramm. Denn nach wie vor bestimmen Grenzkontrollen oder die Politikmaßnahmen gegenüber Migranten innerhalb der Wohlfahrtstaaten den Zugang zu Rechtsansprüchen auf soziale Leistungen. So gibt es zum Beispiel Abstufungen im Zugang je nach Art der Aufenthalts- und Arbeitserlaubnis. ${ }^{24}$ Es ist der Bedarf an Arbeitskräften, der die Handhabung von Arbeitserlaubnissen und damit die Migration beziehungsweise Mobilität aus mittelund osteuropäischen Staaten nach Süd- und Westeuropa beeinflusst, die nach dem Beitritt neuer Mitgliedstaaten im Jahre 2004 noch einmal zunahm. In Ländern mit hoher Nachfrage nach Betreuungskräften, in denen der Arbeitsmarkt für Haushalt und Pflege relativ dereguliert ist, beispielsweise in Großbritannien und südeuropäischen Mitgliedstaaten, waren Migrationskontrollen weniger stark ausgeprägt. Ähnliches gilt entsprechend für männlich geprägte Sektoren wie das Bauwesen.

Um die (Re-)Produktion von Ungleichheiten zu erfassen, lautet hier die Grundannahme, dass die Suche nach den entscheidenden sozialen Mechanismen einen wichtigen Schritt in Richtung auf eine kausale Rekonstruktion von ungleichheitsrelevanten Prozessen erlaubt. Der Begriff »sozialer Mechanismus « bezieht sich auf wiederkehrende Prozesse oder Verläufe und verknüpft identifizierbare Ausgangsbedingungen mit spezifischen Ergebnissen. Zur Darstellung der Prozesse der Reproduktion und Generierung von Ungleichheiten kann zwischen den Typen sozialer Mechanismen und den Analyseebenen unterschieden werden (Abbildung 1). Bei den verschiedenen Typen von sozialen Mechanismen handelt es sich um Mechanismen

23 EU 2013. Abhängige Selbstständigkeit ist eine Arbeitsbeziehung, in welcher der Arbeiter formal selbstständig ist, jedoch unter Bedingungen, die denen abhängig Beschäftigter vergleichbar sind. Neben dem Bausektor finden sich derartige Konstruktionen vor allem im Transportwesen, im Versicherungsgewerbe, bei Dienstleistungen aller Art und in der Werbeindustrie.

24 Siehe etwa Sainsbury 2010. 
wie Exklusion, Hierarchisierung, Chancenhortung und Ausbeutung 25 und damit verknüpften konkreten Praktiken. Bei der Analyse dieser Mechanismen kann annäherungsweise zwischen Mechanismen unterschieden werden, die auf einer mittleren Ebene - Kleingruppen und Netzwerke von Personen - oder auf einer gesamtgesellschaftlichen Ebene - gesellschaftliche Institutionen - angesiedelt sind. Institutionelle Mechanismen der mittleren Ebene bestimmen die Verbindungen zwischen Menschen, Gruppen und interpersonellen Netzwerken, die Beziehungen zwischen einzelnen und kollektiven Akteuren. Die institutionellen Mechanismen der gesamtgesellschaftlichen Ebene beziehen sich auf die Bedeutung von Strukturen und deren (neu auftretende) Eigenschaften für die Entstehung sozialer Ungleichheiten. Konkret liegt aus Sicht der mittleren Ebene der Fokus auf den Effekten von grenzüberschreitender Mobilität für die "Zurückgebliebenen « in den Heimatregionen. Ethnografische Untersuchungen in verschiedenen Teilen der Welt - etwa Osteuropa, Lateinamerika, Afrika und Südostasien - lassen darauf schließen, dass auswandernde Frauen, die einer Beschäftigung in einem anderen Land nachgehen, ihre Kinder meist der Obhut von Ersatz- beziehungsweise Großmüttern und dem weiteren Familienkreis, weniger häufig den Vätern überlassen. ${ }^{26}$ Allerdings ist bisher nicht ausreichend bekannt, welche Mechanismen in der Pflege- und Betreuungsarbeit in Einwanderungs- und Auswanderungsländern Ungleichheiten reproduzieren und erzeugen. Im Bausektor sind es vorwiegend Firmen oder Makler, die als Transmissionsriemen wirken. Dabei arbeiten seit Ende der 1990er Jahre vermehrt nicht klassische Arbeitnehmer ${ }^{27}$, sondern Arbeitskräfte in der rechtlichen Form Selbstständiger. Gerade die formalrechtlich Selbstständigen, die dem Weisungsrecht anderer unterliegen, arbeiten unter prekären Bedingungen und können kaum kontinuierlich für die soziale Sicherung ihrer Familienangehörigen im Herkunftsland sorgen.

Im Hinblick auf Exklusion bezeugen die Mitgliedschaft in Gruppen und die Partizipation an Netzwerken den Grad an Zugehörigkeit, durch den zum Beispiel der Zugang zu Arbeitsplätzen, Wohnraum und Kinderbetreuung erleichtert beziehungsweise erst ermöglicht wird. Für Migranten im Dienstleistungssektor ist die Zugehörigkeit zu Cliquen und Gruppen nicht nur entscheidend bei der Suche nach Arbeit, sondern auch nach Möglichkeiten für die Kinderbetreuung, um sich aus der Ferne um die Kinder zu Hause beziehungsweise in Fällen, in denen ältere Verwandte zurückgeblieben sind, um deren Pflege in den Herkunftsländern zu kümmern. ${ }^{28}$ Auf der nationalgesellschaftlichen Ebene sind Einwanderungspolitiken und dementsprechend die Staatsangehörigkeit entscheidend für die Frage der Zugehörigkeit. Die rechtlich geregelte Zuordnung zu einem Staat und die damit verbundene Vollmitgliedschaft im Sinne gleicher Rechte für Staatsbürger (beispielsweise soziale

$25 \mathrm{Zu}$ weiteren Mechanismen siehe Diewald, Faist 2010.

26 Siehe unter anderem Parreñas 2005; Lutz 2010.

27 Faist et al. 1999. Werkvertragsarbeitnehmer sind Beschäftigte von Subunternehmen, wobei diese Unternehmen im Rahmen der Dienstleistungsfreiheit in der EU oder im Rahmen bilateraler Vereinbarungen mit Drittstaaten einen Auftrag für einen Generalunternehmer ausführen.

28 Piperno 2007.

Leviathan, 41. Jg., 4/2013 
Abbildung 1: Soziale Mechanismen und die Genese sozialer Ungleichheiten (Beispiele)

\begin{tabular}{|c|c|c|}
\hline $\begin{array}{r}\text { Soziale } \\
\text { Formation }\end{array}$ & $\begin{array}{l}\text { Mittlere Ebene } \\
\text { - Kleingruppen \& Netzwerke - }\end{array}$ & $\begin{array}{l}\text { Gesamtgesellschaftliche Ebene } \\
\text { - Gesellschaftliche Institutionen - }\end{array}$ \\
\hline Exklusion & $\begin{array}{l}\text { Zugehörigkeit (z. B. Zugang zu } \\
\text { Netzwerken und Arbeitsplät- } \\
\text { zen) }\end{array}$ & $\begin{array}{l}\text { Einwanderungspolitik \& Staats- } \\
\text { angehörigkeit (z. B. irregulärer } \\
\text { Status, Zirkulation mit Visums- } \\
\text { pflicht) }\end{array}$ \\
\hline Hierarchisierung & $\begin{array}{l}\text { Einstufung von Tätigkeiten als } \\
\text { qualifiziert oder unqualifiziert }\end{array}$ & Massenmediale Skandalisierung \\
\hline Chancenhortung & Unterstützungsnetzwerke & $\begin{array}{l}\text { Opportunitätsstrukturen in } \\
\text { Arbeitsmärkten }\end{array}$ \\
\hline Ausbeutung & $\begin{array}{l}\text { Machtasymmetrien } \\
\text { (z. B. Hausarbeit) }\end{array}$ & $\begin{array}{l}\text { Negative Umverteilung (unge- } \\
\text { rechter Tausch): Abwanderung } \\
\text { von Fach- und Pflegekräften } \\
\text { (»brain drain «); Überweisungen } \\
\text { von Herkunfts- in Zielregionen }\end{array}$ \\
\hline
\end{tabular}

Rechte) und Unionsbürger (etwa die Reise- und Niederlassungsfreiheit in Mitgliedstaaten der EU) sind wichtig, da sie grenzüberschreitende Reisen und Aufenthalte in einem anderen Mitgliedsland erleichtern und somit einen höheren Grad an Transnationalität in Form von grenzüberschreitender Mobilität absichern. Für eines der wichtigsten Herkunftsländer von Pflegekräften, Rumänien, fiel mit dem Eintritt in die EU 2002 die Visumspflicht für Kurzaufenthalte weg, und zugleich wurden weitere Hindernisse abgebaut. Ein anderes Herkunftsland für Pflegekräfte, die Ukraine, ist jedoch ein Drittstaat, mit all den Folgen für die Transnationalität. Staatsbürgerschaft in einem Mitgliedsland der EU und die damit verbundene Reisefreiheit veränderten also die Situation für die Mobilität der angeheuerten Pflegekräfte erheblich. Rumänische Minderjährige besuchen ihre Mütter, die als Pflegekräfte in Italien arbeiten, häufiger als die ukrainischen Kinder deren Mütter, was sich erwartungsgemäß auf das Vertrauensverhältnis zwischen Müttern und Kindern auswirkte. Darüber hinaus ist der Beschäftigungsstatus der rumänischen weiblichen Arbeitskräfte als EU-Bürger von höherer Rechtssicherheit geprägt im Vergleich zu dem der ukrainischen Frauen. Das bedeutet im Hinblick auf die Frage der Ungleichheit, dass Migranten aus Rumänien und der Ukraine in Italien unterschiedliche Optionen in Bezug auf Reisefreiheit oder Zugang zu sozialen Dienstleistungen haben. Die Ungleichbehandlung von Bürgerinnen und Nicht-Bürgerinnen der EU ist ein in poli- 
tischen Debatten in Europa häufig thematisierter Punkt. ${ }^{29}$ Der Rechtsstatus nach Territorialität hat ungleichheitsrelevante Wirkungen etwa im Hinblick auf Transnationalität, aber auch auf Ressourcen wie Zeit, die für die Betreuung der eigenen Familienangehörigen aufgebracht werden kann, wenn sie etwa für langes Anstehen vor den Konsulaten verwendet werden muss.

Gleichzeitig werden hier die Grenzen des Rechtsstatus als Heterogenitätsmerkmal zur Erklärung und zum Verständnis von Ungleichheiten sichtbar. Trotz der Aufhebung der Zugangsbeschränkungen zu Arbeitsmärkten in der EU hat sich die Arbeitsmarktsituation von mobilen Bürgern aus Rumänien, Bulgarien oder Polen in südeuropäischen Mitgliedsländern nicht entscheidend verbessert, was etwa die Arbeitsbedingungen (häufig im informellen Sektor) oder das Einkommen betrifft. Es gibt also auch Hierarchisierungen innerhalb der Gruppe der Arbeiter, die aus EULändern kommt. Die Arbeitschancen der Bürger dieser Staaten sind etwa in Italien oder Spanien vor allem auf als unqualifiziert eingestufte und niedrig bezahlte Stellen beschränkt. Dies stellt eine Art Umwertung von »Mobilen «, also grenzüberschreitend Beschäftigten aus EU-Ländern, in "Migranten ", also extracommunitari, dar und trifft für Beschäftigte im Pflege- und Haushaltsbereich, im Bausektor und in vielen anderen Produktions- und Dienstleistungen gleichermaßen zu. ${ }^{30}$ Diese Beschäftigten fungieren ebenso wie viele extracommunitari als eine Art flexible und disponible »Reservearmee .

Einen weiteren grundlegenden Mechanismus stellt die Hierarchisierung dar. Insbesondere die Bewertung und damit die Einstufung von Pflege- und Hausarbeit als »unqualifiziert « ist der entscheidende Angelpunkt dieses Mechanismus. Haushaltsund Pflegearbeiten sind in der Regel mit unqualifizierter und geschlechtsspezifischer Arbeit assoziiert, nicht zuletzt auch, weil sie in den Herkunftsregionen noch nicht so flächendeckend kommodifiziert sind. Jedoch sind viele der Frauen, etwa aus Osteuropa oder den Philippinen, die in Europa diese Arbeit verrichten, qualifizierte Fachkräfte, die in den Herkunftsregionen eine Fachausbildung absolvierten; zum Beispiel finden sich unter den Frauen aus der Ukraine, die derartige Dienstleistungen in west- und südeuropäischen Ländern erbringen, viele ausgebildete Pflegekräfte wie etwa Krankenschwestern. ${ }^{31}$ In etwas abgeänderter Form lässt sich dieses Muster auch im Bausektor erkennen. Gerade auf Großbaustellen sind im Rahmen einer organisationsorientierten Produktionsweise, bei denen sich Generalunternehmer von einem eigenen Stamm an Facharbeitern unabhängig machen wollen, Subunternehmer (aus anderen Staaten) gefragt, die ausgebildete Fachkräfte einsetzen. Es handelt sich also im Allgemeinen nicht um unqualifizierte Arbeitskräfte, die sich dieser grenzüberschreitenden Lebensform unterwerfen, und die Einstufung erweist sich damit als eine Form der Aufrechterhaltung der Hierarchien.

Auf gesamtgesellschaftlicher Ebene spielen für die hier beschriebene Hierarchisierung nicht nur rechtliche Unterscheidungen eine Rolle, sondern auch semantische

29 Bauböck et al. 2006, S. 85-104.

30 Recchi, Triandafyllidou 2010, S. 141.

31 Piperno 2007.

Leviathan, 41. Jg., 4/2013 
Mechanismen der massenmedialen Skandalisierung. So wurde in den letzten Jahren insbesondere die Abwesenheit von Müttern, die als Migrantinnen in west- und südeuropäischen Ländern arbeiten, thematisiert. Zeitungen und Zeitschriften berichteten über »Euro-Waisen « (Euro-orphans) oder "Sozialwaisen " (social orphans), deren »schlechte « Mütter beschlossen, in den Westen zu gehen, um durch Arbeitstätigkeit persönliche Erfüllung zu suchen, ohne die negativen Auswirkungen auf ihre schutzlosen Kinder zu berücksichtigen. ${ }^{32}$ Diese Kategorisierungen haben semantische Klassifizierungen im Hinblick auf den sozialen Status zur Folge, in diesem Falle "guter « oder »schlechter « Elternschaft. Die empirische Forschung lässt hier differenziertere Bewertungen zu, denn neben der Tatsache der familiären Trennung gibt es im Rahmen von »transnationaler Mutterschaft « beziehungsweise »transnationaler Vaterschaft « die verschiedensten Strategien, die Leiden dieser Trennung abzumildern, von gelegentlichen Telefonaten und Besuchen im Herkunftsland bis hin zum Nachholen der Kinder und anderer Angehöriger in das Immigrationsland. ${ }^{33}$ Auch weisen die Praktiken vieler weiblicher Pflegekräfte oder männlicher Bauarbeiter, die in West- und Südeuropa arbeiten, einen hohen Grad an Transnationalität im Hinblick auf Kommunikationsdichte auf, um mit ihren Familien und insbesondere den Kindern in Kontakt zu bleiben. Ein Großteil der Forschung verweist allerdings darauf, dass die in den Herkunftsländern verbliebenen neuen Betreuungspersonen wie etwa die Großmütter selbst oft überlastet sind. Die Pflegesituation des Teils der transnational verstreuten Familie im Zielland ist häufig ebenfalls prekär: Während die Ausgewanderten für Pflege und Betreuung im Arbeitgeberhaushalt sorgen, bleibt die Betreuung ihrer eigenen Kinder auf der Strecke, weil es die Arbeitsbedingungen nicht erlauben, sich um eben diese zu kümmern. ${ }^{34}$

Soziale Schließung im Sinne von Privilegierung als Mittel, um die eigenen Chancen zu erhöhen, wird von manchen (ethnischen, religiösen, verwandtschaftsbezogenen) Gruppen gelegentlich benutzt, um bestimmte Nischen zu besetzen, wie beispielsweise im wirtschaftlichen Bereich im Gastronomiegewerbe eines Stadtviertels. ${ }^{35}$ Ein Beispiel wäre eine Gruppe rumänischer Krankenpflegerinnen, wenn sie sowohl eine räumliche als auch eine berufliche Nische in der Altenpflege in einer italienischen Stadt insgesamt oder einem Stadtteil besetzen. So werden neue Migranten aus der eigenen Region entweder für andere Familien angeworben oder um diejenigen zu ersetzen, die zurück nach Osteuropa ziehen. In einem solchen Fall stellt das Mittel der bevorzugten Einbeziehung der eigenen Leute - ein Mechanismus der Chancenhortung - sicher, dass die Mitglieder einer Gruppe (zum Beispiel aus der gleichen Heimatstadt) oder eines Unterstützungsnetzwerks von solchen Empfehlungen profitieren. Es ergeben sich daraus zum einen ungleichheitsrelevante Kategorisierungen in Bezug auf den Zugang zu Arbeitsplätzen und damit unter anderem auf die Einkommen für Mitglieder anderer Gruppen. Aber zugleich handelt es sich auch um

32 Pustułka 2012, unter Rückgriff auf Sylwia Urbańska.

33 Vgl. Dreby, Adkins 2010.

34 Pérez Orozco 2009.

35 Tilly 2005, S. 153-170. 
einen Schutz (wie es Karl Polanyi beschrieben hat): Angesichts der Kommodifizierung der Arbeit können sich die Arbeiter(innen) allein durch Zusammenschluss gewisse Lebenschancen und tarifliche Verbesserungen erkämpfen, dazu benötigen sie Zusammenhalt, der am sichersten gewährleistet ist durch Kolleg(inn)en aus ihrer Heimat. ${ }^{36}$ Insgesamt bilden sowohl Chancenhortung als auch Ausbeutung (siehe unten) soziale Mechanismen, die zu Monopolbildung führen. Verteidigt werden solche Monopole dann beispielsweise mit Zuschreibungen wie Stereotypisierungen, indem die Unterworfenen etwa als »faul « oder » ungebildet « kategorisiert werden. Chancenhortung geht häufig mit Ausbeutung einher, so beispielsweise, wenn Verwandte im Rahmen von gesponserter Immigration in Zwangsarbeitsverhältnissen gehalten und gezwungen werden, die Kosten für die Immigration abzuarbeiten.

Im italienischen Sozialsystem ist die informelle Anwerbung eine Möglichkeit für Frauen aus mittel- und osteuropäischen Ländern, sich Chancen zu eröffnen. Das italienische Sozialsystem bietet etwa im Gegensatz zum deutschen keine formell geregelten Pflegeangebote durch Institutionen wie in Deutschland die Pflegeversicherung und führt von daher zu informellen Pflegearrangements ohne gesetzlichen Rahmen und tarifvertragliche Regelungen. ${ }^{37}$ In der Folge kann die Entwicklung neuer sozialer Sicherungsgefüge für die Altenpflege in Italien festgestellt werden, die jedoch nicht so hochgradig differenziert sind wie formale nationale Systeme der sozialen Sicherung. Solche grenzübergreifenden Sozialgefüge können im Zusammenhang mit den rechtlich-institutionellen Vorgaben als Teil der Opportunitätsstrukturen für die Beschäftigung von Pflegekräften gesehen werden.

Im Hinblick auf grenzübergreifende Strategien sozialer Sicherung ist der Grad an Transnationalität also von Bedeutung: ${ }^{38}$ Bei einem hohen Grad an Transnationalität - also bei hoher Dichte der grenzübergreifenden Kontakte, einer Vielzahl regelmäßiger Sendungen von Waren und Geld, vielen Auslandsbesuchen und auch bei mehrfacher Staatsangehörigkeit - ist die Wahrscheinlichkeit hoch, dass private soziale Sicherung innerhalb von Familien und Freundschaftscliquen grenzübergreifend organisiert ist, während bei einem geringeren Grad an Transnationalität lokale Netzwerke im Zielland eine stärkere Rolle spielen. Diese Vermutung wird durch eine detaillierte Analyse der Strategien sozialer Sicherung bei verschiedenen Kategorien von grenzüberschreitend Beschäftigten in den transnationalen Räumen Türkei-Deutschland (beispielsweise Arbeitsmigranten und Flüchtlinge), Polen-Deutschland (etwa EU-Migranten und Aussiedler) und Kasachstan-Deutschland (zum Beispiel Spätaussiedler) in den Feldern Altersversorgung, Gesundheit, Bildung und Kinderbetreuung bestätigt. ${ }^{39}$ Transnational orientierte Formen sozialer Sicherung äußern sich in konkreten sozialen Mechanismen wie zeitweiliger Übertragung der Pflege, zum Beispiel wenn Eltern ihre im Land der Beschäftigung geborenen Kinder

36 Am Beispiel eines Streiks von »sans papiers« siehe Barron et al. 2011.

37 Vgl. Bettio et al. 2006.

38 Siehe Ergebnisse des Teilprojekts »Transnationalität und die ungleiche Verteilung informeller sozialer Sicherung«"im SFB 882 »Von Heterogenitäten zu Ungleichheiten« (www.sfb882.uni-bielefeld.de/de/projects/c3).

39 Amelina et al. 2012. 
für längere Perioden im Herkunftsland in die Obhut von Verwandten geben, etwa für einen Schulbesuch, und somit soziale Kontrolle ausüben. Ungleichheit erhaltend sind derartige Praktiken deshalb, weil sie die betroffenen Kinder in ihrer Eigenständigkeit und damit in ihren Entwicklungsmöglichkeiten einschränken. Mit Blick auf die eben genannten transnationalen Räume ist das vor allem im Raum DeutschlandTürkei zu beobachten, der insgesamt durch einen relativ hohen Grad an Transnationalität im Vergleich zu den beiden anderen transnationalen sozialen Räumen gekennzeichnet ist. Im Falle Deutschland-Polen - moderate Transnationalität - sind Praktiken zur Aufrechterhaltung des familiären Zusammenhangs eher durch Sommerurlaube in einem der beteiligten Länder geprägt, während im Falle DeutschlandKasachstan - niedrige Transnationalität - derartige Praktiken weitgehend fehlen. Schließlich impliziert im letzteren Fall das Abwandern ganzer Familien- und Verwandtschaftsgruppen, dass wenige Anknüpfungspunkte in den Herkunftsländern verblieben.

Beim Mechanismus der Ausbeutung verletzen aus Sicht der Beschäftigten die Arbeitgeber auf der Grundlage von Machtasymmetrien der Beteiligten Normen über faires und zulässiges Handeln. Aus relationaler Sicht verzichten die Beschäftigten bei irregulären Arbeitsverhältnissen in Haushalten, die mitunter sogar ohne offizielle Aufenthaltserlaubnis zustande kommen, weitgehend auf die Möglichkeit, eventuelle Ansprüche auf dem Rechtsweg durchzusetzen, da die oder der Beschäftigte sich in dem Dilemma befindet, entweder im informellen Sektor ausgebeutet zu werden oder ansonsten fürchten muss, wegen seines rechtswidrigen Aufenthalts ausgewiesen zu werden, selbst wenn ein Gericht den Arbeitgeber zur Verantwortung ziehen würde. Hieraus entsteht eine Verstärkung der Machtasymmetrie zwischen den entsprechenden Unternehmern und den Arbeitern im Sinne rechtlicher und sozialer Verhältnisse. Dass Gleichheitsnormen wie etwa die Tarifgleichheit, die in etlichen Konventionen der Internationalen Arbeitsorganisation festgehalten sind, hier verletzt werden, führt zur Thematisierung derartiger Arbeitsverhältnisse in öffentlichen Foren wie der Internationalen Arbeitskonferenz. ${ }^{40}$ Es zeigt sich, dass es sektorale Unterschiede in der Ungleichheit gibt: So sind etwa Kontrollen von arbeitsrechtlichen Vorschriften in Haushalten oder auf Baustellen ungleich schwerer durchzuführen als in festen Betriebsstätten. Und es gibt Unterschiede zwischen den europäischen Ländern: In stark deregulierten Staaten wie Spanien dienen beispielsweise der Pflege- und der Bausektor als wichtige Zugangsschleusen zum formalen Arbeitsmarkt; diese Funktion wird auch verstärkt durch sogenannte Regularisierungskampagnen, mit denen Migranten mit irregulärem Rechtsstatus integriert werden. ${ }^{41}$ Daraus ergibt sich, dass in einem wichtigen Wachstumsbereich der Dienstleistungen der Mechanismus der Ausbeutung an Relevanz gewinnt.

Eine genauere Analyse des Mechanismus der Ausbeutung deutet darauf hin, dass soziale Ungleichheiten in der Regel nicht auf eine einzige Dimension der Heterogenität bezogen sind. In den Haushalten der Arbeitgeber nimmt die Ungleichheit im Hinblick auf Ressourcen, Status und Macht beispielsweise zu, weil Migranten als

40 ILO 2010.

41 Leon 2010. 
Hausangestellte hinzukommen. Durch die Beschäftigung von Migranten nehmen die Ungleichheiten zwischen den Geschlechtern in Haushalten nicht ab, da die Arbeitsteilung zwischen Männern und Frauen aufgrund der Anwesenheit ausgebeuteter, vornehmlich weiblicher Migranten zwar verändert wird - so können Frauen aus der Mittelschicht dank der Unterstützung durch ausländische Hausangestellte in die Lage versetzt werden, ihre beruflichen Laufbahnen konsequenter zu verfolgen und damit die geschlechtsspezifischen Muster der Teilhabe an formalen Arbeitsmärkten aufbrechen ${ }^{42}$-, aber gleichzeitig durch diese Hausangestellten neue Ungleichheitsmuster eingebaut werden. Über transnationale Prozesse sind in den Immigrationsländern sowohl die Produktion von Ungleichheiten in Haushalten und die Verbesserung der Stellung von Frauen in Deutschland oder Italien in den formalen Arbeitsmärkten miteinander gekoppelt.

Die Ausbeutung zeigt sich auf gesamtgesellschaftlicher Ebene in einer Umverteilung zwischen den Herkunfts- und Zielregionen, die in beide Richtungen verläuft. Erstens ist die Abwanderung von Pflegekräften von Osteuropa nach West- und Südeuropa festzustellen, das heißt in den Zielländern sind etliche der in Privathaushalten tätigen Arbeitskräfte in den Herkunftsländern ausgebildete Krankenpflegerinnen. Sinngemäß ergibt sich in anderen Sektoren wie der Bauwirtschaft eine Wanderungsbewegung »von Köpfen und Muskeln «. Infolgedessen gehen Investitionen in die Berufsausbildung verloren, und in den Herkunftsregionen kommt es zu einem Arbeitskräftemangel in den betroffenen Bereichen. Um den gesamten Prozess darzustellen, müssen die Anreize für junge Menschen einbezogen werden, die sich durch das Vorbild erfolgreicher berufstätiger Fachkräfte im Ausland zu einer Ausbildung entschließen, ${ }^{43}$ sowie die Folgen der Re-Migration und der Überweisungen von Geld in die Herkunftsländer. Es ist einleuchtend, dass dies für diejenigen Auswanderungsregionen dann zu einem Verlust führt, wenn die abgewanderten Fachkräfte nicht durch vermehrte Ausbildung von geeignetem Nachwuchs oder die Anwerbung ausländischer Arbeitskräfte ersetzt werden können, was man als Verlustkaskaden bezeichnen könnte. Dies ist bis zu einem gewissen Grade in den Ländern der Fall, in welche wiederum keine Arbeitskräfte einwandern, sondern aus denen nur Fachkräfte auswandern, beispielsweise die zentralasiatischen Länder wie Kirgisistan oder Usbekistan. Am Ende der Kaskade entsteht eine Lücke, die nicht mehr durch inoder ausländische Arbeitskräfte aufgefüllt werden kann.

Zweitens gleichen auf regionaler Ebene die Remissionen als Ausdruck von Transnationalität aus dem Ziel- ins Herkunftsland die erwähnten Verluste nicht aus. Konkret gibt es keine Hinweise darauf, dass Ungleichheiten zwischen Haushalten gerade in den Herkunftsregionen abnehmen würden, zumindest nicht kurzfristig auch wenn es Hinweise auf Armut reduzierende Wirkungen von Überweisungen auf Haushaltsebene gibt. ${ }^{44}$ Schließlich sind in den Emigrationsregionen nicht alle Haushalte an internationaler Migration beteiligt. Und auf diejenigen, die migrieren, üben

42 Nakano Glen 1992.

43 Stark 2004.

44 Skeldon 1997.

Leviathan, 41. Jg., 4/2013 
ihre soziale Klasse und die Vernetzung über Migrationsnetzwerke einen großen Einfluss auf die Art ihrer Mobilität aus. Diese Stratifizierung, bei der auch die Zwischenkategorien jenseits von Hochqualifizierten einerseits und marginalisierten Arbeitsmigranten andererseits berücksichtigt werden müsste, ${ }^{45}$ impliziert, dass in den Emigrationsregionen nicht alle in gleichem Maße von den Remissionen profitieren. Sicker- und Multiplikator-Effekte von finanziellen Transfers sind über die beteiligten Haushalte hinaus nur schwer nachzuweisen. Im Hinblick auf Remissionen sind es die Regierungen der Herkunftsländer, die einen Vorteil daraus ziehen: Weltweit wird fast die Hälfte aller Remissionen über eine Bank und in der Währung der Beschäftigungsländer an die Notenbank des Herkunftslands überwiesen, die wiederum der Bank der Familie das Geld in der Währung des Emigrationslands auszahlt. ${ }^{46}$

Diese Beobachtung führt zur Frage, ob soziale Ungleichheiten auch in der anderen Richtung entstehen. Denn tatsächlich werden auch Ressourcen aus den Emigrations- in die Immigrationsregionen umverteilt: Neben der Abwanderung der Fachkräfte selbst gibt es ebenso umgekehrte finanzielle Überweisungen zu verzeichnen, also monetäre Transfers von den Herkunfts- in die Zielregionen. Migranten müssen oft beträchtliche Summen aufwenden, um sich in den Zielregionen niederzulassen, beispielsweise für ein Studium oder das Einklagen einer Arbeits- und Aufenthaltserlaubnis. Es überrascht nicht, dass diese Ungleichheiten insbesondere von politischen und wissenschaftlichen Vertretern der Emigrationsregionen als Hinweis auf einen ungerechten Tausch im weitesten Sinne vorgebracht werden. ${ }^{47}$

Um Mechanismen der Produktion von Ungleichheiten umfassend zu verstehen, ist das Zusammenwirken der verschiedenen Ebenen zu betrachten. Die ungleichheitsrelevanten Konsequenzen des Rechtsstatus müssen daher immer im Zusammenhang mit den (Re-)Produktionsbedingungen in den beiden Ländern gesehen werden. So gab es Mitte der 1990er Jahre auf deutschen Baustellen sowohl Werkvertragsarbeitnehmer aus mittel- und osteuropäischen Ländern als auch aus EUMitgliedsländern, allen voran Portugal und Irland; fast ein Viertel der Bauarbeiter Portugals arbeitete seinerzeit im Ausland. Grenzübergreifende Werkverträge waren und sind in einigen Sektoren der Bauwirtschaft gang und gäbe. Die Produktionsprozesse auf Baustellen sind häufig durch enge Abstimmungen ${ }^{48}$ gekennzeichnet, was gerade auf Großbaustellen angesichts der Notwendigkeit der Zusammenarbeit einer großen Zahl von Akteuren bedeutet, dass Hierarchien flach sind und Personal möglichst flexibel vor Ort eingesetzt werden muss. Um die Gewinnspanne zu steigern und dabei Aufträge fristgerecht erfüllen zu können und beispielsweise nicht mit

45 Siehe auch Verwiebe 2004.

46 Vor diesem Hintergrund ist auch die Initiative der Weltbank zu verstehen, die Anfang der 2000er Jahre das Thema Remissionen auf die internationale Agenda setzte. Es geht dabei um die Kontrolle von Überweisungen durch internationale Organisationen und nationale Regierungen angesichts der Tatsache, dass über die Hälfte der von Migranten ausgehenden Finanztransfers nicht durch Banken getätigt wird.

47 Khadria 2009.

48 Perrow 1986. 
Konventionalstrafen belegt zu werden, ist der Anreiz für Bauunternehmer - in diesem Falle der Subunternehmer - relativ hoch, irreguläre oder doch zumindest "graue« Beschäftigungsverhältnisse zu fördern. Die Senkung der Lohnkosten ist hier ein probates Mittel, da andere Kostenfaktoren wie Maschinen oder Baustoffe kaum Spielraum für Einsparungen gewähren

\section{Zusammenfassung und offene Fragen}

Eine Annäherung an die transnationale soziale Frage beginnt damit, die Verbindungen von Personen und Gruppen zwischen verschiedenen Orten und zwischen verschiedenen Staaten zu kartieren und die in grenzübergreifenden Transaktionen wirksamen sozialen Mechanismen der Ungleichheitsproduktion zu bestimmen. Ein vorläufiges Fazit zu Arbeit und sozialer Sicherung in transnationalen sozialen Räumen in Europa ergibt, dass die Anstrengungen von Migranten und ihren Angehörigen häufig alte Ungleichheiten verstärken und neue hervorrufen. Obwohl Strategien der grenzübergreifenden sozialen Sicherung die individuellen beziehungsweise familiären Existenzgrundlagen erheblich verbessern können, zum Beispiel durch Überweisungen für Ausbildung, Gesundheitsversorgung oder Konsum, bleiben grundlegende Ungleichheiten zwischen Herkunfts- und Zielregionen in der Regel erhalten. Institutionell verankerte Formen der öffentlichen sozialen Sicherung in den Herkunftsregionen sind häufig zusätzlichem Druck etwa durch die Abwanderung von qualifizierten Fachkräften ausgesetzt. Es entstehen neue Ungleichheiten im Verlauf von grenzübergreifender Migration wie beispielsweise Unterschiede zwischen Haushalten mit oder ohne Remissionen in die Herkunftsregionen oder die erwähnten Unterschiede bei der geschlechtsspezifischen Teilung der Arbeit in den Immigrationsregionen, wenn die Emanzipation der Frauen des Immigrationslands dazu führt, dass Frauen aus den Peripherieländern die vakant gewordene Stelle für Pflegedienste im Haushalt übernehmen.

Eine weitere Bearbeitung dieser Fragestellung ist ein dringliches Problem sozialwissenschaftlicher Forschung. Denn die Öffnung der Ländergrenzen für den gemeinsamen Markt in der EU im Sinne einer "negativen Integration " wurde in den letzten vier Jahrzehnten eben nicht von einer " positiven Integration " und der Schaffung einheitlicherer Sozialstandards begleitet. ${ }^{49}$ Gleichzeitig sind Konvergenzen etwa im Pflege- und Haushaltsbereich unübersehbar: Beispielsweise schritt im letzten Jahrzehnt die Kommodifizierung und teilweise Informalisierung der Pflegearbeit nicht nur in stark deregulierten Staaten wie Großbritannien und Spanien unaufhaltsam fort, sondern auch in (bisher) hoch regulierten Wohlfahrtsstaaten wie Dänemark und Schweden. ${ }^{50}$ Deutlich ist dies also innerhalb eines Sektors, der in einem bestimmten Segment nur wenige attraktive Arbeitsplätze zu vergeben hat, die von hohen körperlichen Belastungen gekennzeichnet sind und deshalb von Geringqualifizierten besetzt werden, deren Marktmacht in der Regel schwach ist. Innerhalb

49 Streeck 1995.

50 Williams 2012.

Leviathan, 41. Jg., 4/2013 
des liberalen Paradigmas der EU expandieren mit der Marktöffnung die grenzüberschreitenden Beschäftigungsformen, zusätzlich angefeuert durch die verbreiteten negativen Visionen der demographischen Entwicklung der europäischen Gesellschaften. Eine Expansion von öffentlichen Pflegeeinrichtungen - eine Option, die noch zu Zeiten des keynesianischen Wohlfahrtsstaats der 1960er und 1970er Jahre im Mittelpunkt gestanden hätte - ist bislang nicht zu beobachten. Im Rahmen der herrschenden Fiskal- und Finanzpolitik der EU ist der Einzug von Elementen einer supranationalen Sozialpolitik nicht möglich. Dies bedeutet auch, dass die hier geschilderten sozialen Mechanismen der Ungleichheitsproduktion in ihrer Wirkung voraussichtlich nicht abgemildert, sondern in Zukunft noch weiter verstärkt werden.

Aus der empirischen Analyse ergibt sich eine Reihe weiterführender Fragen für zukünftige Forschungen, von denen zwei im Hinblick auf die transnationale soziale Frage zentral sind:

Erstens ist neben einer Ausweitung der empirischen Analyse über Haushalts- und Pflegearbeit und den Bausektor hinaus auf anderen Feldern die subjektive Bedeutung und Zuschreibung von sozialen Ungleichheiten näher zu erfassen. Denn eine transnationale soziale Frage ist nur vor dem Hintergrund der als normativ ungerechtfertigt eingestuften sozialen Ungleichheiten denkbar. Die Maßstäbe für die Bewertung von Ungleichheiten müssen an Normen ausgerichtet sein, die relativ beziehungsweise relational sind und Vergleiche mit Kategorien von Personen zulassen, die sozial gesehen nicht zu weit voneinander entfernt sind. ${ }^{51}$ Die soziologisch spannende Frage ist, welche Kategorien von Personen und welche Normen von den beteiligten Akteuren in den jeweiligen Arbeitsorganisationen und in politischen Arenen als relevant eingestuft werden. Die Wahrnehmung von Ungleichheiten im Hinblick auf Ressourcen, Status und Macht kann sich auf die Emigrations-, die Immigrationsseite oder auf beide beziehen. Ungleichheiten sind in transnationaler Perspektive als »entgrenzt " anzusehen: Während die Grenzen von Staaten und insbesondere die Grenzen von Zugehörigkeit und Mitgliedschaft durchaus konstituierend für die Lebenschancen von Menschen wirken, stimmen die sozialen, kulturellen und wirtschaftlichen Grenz(ziehung)en in den Lebenswirklichkeiten der grenzüberschreitend Beschäftigten nicht unbedingt damit überein. Die EU ist ein besonders interessanter Fall, da nicht nur ärmere nationale Staaten Referenzen für die subjektive Einschätzung von Ungleichheiten bilden können, sondern auch die reicheren Wohlfahrtsstaaten - sowohl für die Menschen aus den relativ ärmeren Regionen der EU als auch diejenigen mit außerhalb der EU liegenden Herkunftsregionen. Die sozialen Lebenswelten von Individuen und gegebenenfalls ihre Bewertungsmaßstäbe reichen also über Staatsgrenzen hinweg. Beispielsweise gibt es Hinweise darauf, dass etwa Personen aus Polen, Litauen und Rumänien, die in Westeuropa arbeiten und leben, im Vergleich zur Situation in der Herkunftsregion in Bezug auf Einkommen und Karrierechancen sozial aufsteigen, obwohl sie, gemessen an den Standards in

51 Panning 1983. Ein transnationaler Ansatz liegt durch seine grenzübergreifende Analyse quer zu den Problemen der Messung und politischen Kommunikation von Ungleichheit, die vorrangig entlang von Staatsgrenzen verläuft (vgl. Lepenies 2010). 
den Zielländern, sozial am unteren Ende rangieren, zum Beispiel aufgrund der Nichtanerkennung ihrer im Herkunftsland erworbenen Schul- und Berufsabschlüsse. ${ }^{52}$

Zweitens ist zu prüfen, ob und in welcher Weise die hier aus der Sicht eines Forschers aufgeworfene transnationale soziale Frage in Europa in öffentlichen Foren, Debatten und politischen Konflikten eine Rolle spielt. Während soziale Sicherung im Kontext grenzübergreifender Mobilität zu einem leicht skandalisierbaren Thema werden kann - man denke nur an die erwähnten Berichte über Pflegewaisen oder die Mobilität von Roma in westeuropäischen Ländern -, ist es gerade die internationale Migration, die die EU-Mitgliedstaaten davon abhält, weitergehende supranationale Immigrationsregulierungen für die Wohlfahrt der grenzüberschreitend Beschäftigten zu treffen, solange ein wesentlicher Teil der staatlichen sozialen Sicherung auf der Möglichkeit der Lenkung und der damit verbundenen partiellen Abschottung national regulierter Arbeitsmärkte beruht. Auch wenn eine konsequente Supranationalisierung die Exklusions- und Inklusionsprozesse der (Re-)Produktion von Ungleichheiten gewissermaßen auf eine höhere Stufe - vom Nationalstaat zur EU - verlagern würde, wäre doch innerhalb der EU schon etwas gewonnen. Nationale Staatsbürgerschaft und europäische Bürgerschaft sind gleichermaßen ein Instrument sozialer Schließung nach außen und sozialer Integration nach innen. Die relevante soziologische Frage ist, wie in konflikthaften Prozessen jeweils die Grenzen, verstanden nicht nur im Sinne territorialer, sondern auch mitgliedschaftlicher Zugehörigkeit, verschoben werden.

\section{Literatur}

Amelina, Anna et al. 2012. Ties that protect? The significance of transnationality for the distribution of informal social protection in migrant networks. SFB 882 Working Paper Series 6. Bielefeld. http://sfb882.uni-bielefeld.de/sites/default/files/SFB_882_WP_0006_Amelina_Bilecen_Barglowski_Faist.pdf (Zugriff vom 27.08.2013)

Barron, Pierre et al. 2011. On bosse ici, on reste ici! La grève des sans-papiers: une aventure inédite. Paris: La Découverte.

Bauböck, Rainer et al. 2006. "Nationality in public international law and European law «, in Acquisition and loss of nationality. Volume 1: Comparative analyses, hrsg. v. Bauböck, Rainer et al., S. 35-104. Amsterdam: Amsterdam University Press.

Beck, Ulrich; Proferl, Angelika. Hrsg. 2010. Große Armut, großer Reichtum. Zur Transnationalisierung sozialer Ungleichheit. Frankfurt a. M.: Suhrkamp.

Berger, Peter; Weiß, Anja. Hrsg. 2008. Transnationalisierung sozialer Ungleichheit. Wiesbaden: VS Verlag für Sozialwissenschaften.

Bettio, Francesca; Simonazzi, Annamaria; Villa, Paola 2006. »Changing care regimes and female migration ", in Journal of European Social Policy 16, 3, S. 271-285.

Cabrera, Luis. Hrsg. 2011. Global governance, global government: institutional visions for an evolving world system. Albany: State University of New York Press.

Carver, Terrell; Bartelson, Jens. Hrsg. 2011. Globality, democracy, and civil society. New York: Routledge.

Castles, Stephen; Miller, Mark 2009. The age of migration: international population movements in the modern world. 4. Auflage. Houndmills: Palgrave Macmillan.

52 Favell, Recchi 2011, S. 74.

Leviathan, 41. Jg., 4/2013 
Crevits, Dries; Van Buggenhout, Bea 2005: »Globalisation, worker mobility and social protection ", in Social protection, globalised, hrsg. v. Berghman, Jos et al., S. 24-55. Leuven: Leuven University Press.

De Swaan, Abram 1988. In care of the state: health care, education, and welfare in Europe and America during the modern era. Oxford: Oxford University Press.

Diewald, Martin; Faist, Thomas 2011. »Von Heterogenitäten zu Ungleichheiten: Soziale Mechanismen als Erklärungsansatz der Genese sozialer Ungleichheiten ", in Berliner Journal für Soziologie 21, 1, S. 91-114.

Dreby, Joanna; Adkins, Timothy 2010. »Inequalities in transnational families «, in Sociology Compass 4, 8, S. 673-689.

EU 2013. Social protection rights of economically dependent self-employed workers. Directorate-General for Internal Affairs. Employment and Social Affairs. IP/A/EMPL/ST 2012-02. PE 507.449. Brüssel: European Union.

Faist, Thomas 2000. »Soziale Bürgerschaft in der Europäischen Union: Verschachtelte Mitgliedschaft ", in Die Europäisierung nationaler Gesellschaften. Kölner Zeitschrift für Soziologie und Sozialpsychologie, Sonderheft 40, hrsg. v. Bach, Maurizio, S. 229-250. Wiesbaden: Westdeutscher Verlag.

Faist, Thomas 2009. "The transnational social question: social rights and citizenship in a global context «, in International Sociology 24, 1, S. 7-35.

Faist, Thomas 2012. »Toward a transnational methodology: methods to address methodological nationalism, essentialism, and positionality «, in Revue Européenne des Migrations Internationales 28, 1, S. 51-70.

Faist, Thomas; Fauser, Margit; Reisenauer, Eveline 2013. Transnational migration. Cambridge: Polity Press.

Faist, Thomas et al. 1999. Ausland im Inland. Die Beschäftigung von Werkvertragsabeitnehmern aus Mittel- und Osteuropa in Deutschland. Baden-Baden: Nomos.

Faist, Thomas; Ulbricht, Christian 2014. »Constituting nationality through transnationality: categorizations and mechanisms of inequality in German integration debates ", in Fear and anxiety over national identity, hrsg. v. Foner, Nancy; Simon, Patrick. New York: Russell Sage Foundation (im Erscheinen).

Fauser, Margit et al. 2012. Middling transnationality: an investigation into cross-border involvement among migrants and non-migrants in Germany. Beitrag zur Konferenz »Social Inequality and Transnational Capital«, 9.-10.11.2012, Wissenschaftszentrum Berlin.

Favell, Adrian; Recchi, Ettiore 2011. "Social mobility and spatial mobility ", in Sociology of the European Union, hrsg. v. Favell, Adrian; Guiraudon, Virginie, S. 50-75. Basingstoke: Macmillan.

Fischer-Lescano, Andreas; Möller, Katja 2012. Der Kampf um globale soziale Rechte. Berlin: Verlag Klaus Wagenbach.

Goldin, Ian; Cameron, Geoffrey; Balarajan, Meera 2012. Exceptional people. How migration shaped our world and will define our future. Princeton: Princeton University Press.

Groenendijk, Kees; Hoffman, Holger; Luiten, Maaike 2013. Das Assoziationsrecht EWG/Türkei. Rechte türkischer Staatsangehöriger in der EuGH-Rechtsprechung. Baden Baden: Nomos.

Heidenreich, Martin. Hrsg. 2006. Die Europäisierung sozialer Ungleichheit. Frankfurt a. M.: Campus.

Heidenreich, Martin; Wunder, Christoph 2008. »Patterns of regional inequality in the enlarged Europe", in European Sociological Review 24, 1, S. 19-36.

Hondrich, Karl-Otto 1984. »Der Wert der Gleichheit und der Bedeutungswandel von Ungleichheit", in Soziale Welt 35, 2, S. 267-293.

ILO (International Labour Organization) 2010. Decent work for domestic workers. International Labour Conference, 99th Session 2010, Report IV (1). Genf: ILO.

Khadria, Binod 2009. "Adversary analysis and the quest for global development: optimizing the dynamic conflict of interests in the transnational divide of migration «, in Social Analysis 53, 3, S. 106-122.

Leon, Margarita 2010. "Migration and care work in Spain: the domestic sector revisited ", in Social Policy and Society 9, 3, S. 409-418.

Lepenies, Philipp H. 2010. »Gezählte Armut. Von den methodischen und politischen Tücken, die extreme Armut auf der Welt erfassen zu wollen «, in Leviathan 38, 1, S. 103-118. 
Lutz, Helma 2010. »Gender in the migratory process «, in Journal of Ethnic and Migration Studies 36, 10, S. 1647-1663.

Marshall, Thomas H. 1964 [1950]. Citizenship and social class. Cambridge: Cambridge University Press.

Mau, Steffen 2007. Transnationale Vergesellschaftung. Die Entgrenzung sozialer Lebenswelten. Frankfurt a. M.: Campus.

Morris, Lydia 2002. Managing migration: civic stratification and migrants rights. London: Routledge.

Nakano Glen, Evelyn 1992. "From servitude to service: historical continuities in the racial division of paid reproductive labor ", in Signs: Journal of Women in Culture and Society 18, 1, S. $1-43$.

Panning, William H. 1983. "Inequality, social comparison, and relative deprivation ", in American Political Science Review 77, 2, S. 323-329.

Parreñas, Rhacel S. 2005. Children of global migration: transnational families and gendered woes. Stanford: Stanford University Press.

Pérez Orozco, Amaia 2009. Global perspectives on the social organization of care in times of crisis: assessing the situation. UN-INSTRAW. Gender, migration and development series, Working paper 6. Santo Domingo: UN-INSTRAW.

Perrow, Charles 1986. "Economic theory of organization ", in Theory and Society 15, 1, S. 11-45.

Pfau-Effinger, Birgit 2000. »Conclusion: gender cultures, gender arrangements and social changes in the European context ", in Gender, economy and culture: the European Union, hrsg. v. Duncan, Simon; Pfau-Effinger, Birgit, S. 262-279. London: Routledge.

Piperno, Flavia 2007. "From care drain to care gain: migration in Romania and Ukraine and the rise of transnational welfare ", in Development 50, 4, S. 63-68.

Pustułka, Paulina 2012. »Polish mothers on the move: transnationality and discourses of gender, care, and co-residentiality requirement in the narratives of Polish women raising children in the West ", in Annales Universitatis Paedagogicae Cracoviensis, Studia Sociologica IV, 2, S. $162-175$.

Recchi, Ettore; Triandafyllidou, Anna 2010. »Crossing over, heading West and South: mobility, citizenship and employment in the enlarged Europe", in The changing face of labour migration in Europe, hrsg. v. Menz, Georg; Caviedes, Alexander, S. 125-148. London: Palgrave Macmillan.

Sabates-Wheeler, Rachel; Feldman, Rayah. Hrsg. 2011. Migration and social protection: claiming social rights beyond borders. Houndmills: Palgrave Macmillan.

Sainsbury, Diane 2010. Welfare states and immigrant rights: the politics of inclusion and exclusion. Oxford: Oxford University Press.

Skeldon, Ronald 1997. Migration and development: a global perspective. London: AddisonWesley Pub.

Stark, Oded 2004. »Rethinking the brain drain «, in World Development 32, 1, S. 15-22.

Streeck, Wolfgang 1995. "From market-building to state-building? Reflections on the political economy of European social policy ", in European social policy. Between fragmentation and integration, hrsg. v. Leibfried, Stephan; Pierson, Paul, S. 389-431. Washington: The Brookings Institution.

Tilly, Charles 2005. Identities, boundaries \& social ties. Boulder: Paradigm.

Verwiebe, Roland 2004. Transnationale Mobilität innerhalb Europas. Eine Studie zu den sozialstrukturellen Effekten der Europäisierung. Berlin: edition sigma.

Williams, Fiona 2012. »Converging variations in migrant care work in Europe", in Journal of European Social Policy 22, 4, S. 363-376. 
Zusammenfassung: Welche Konsequenzen haben grenzübergreifende Praktiken der sozialen Sicherung für soziale Ungleichheiten in Europa? Die transnationale soziale Frage ist nicht nur gekennzeichnet durch eine Thematisierung von Ungleichheiten entlang von Heterogenitäten wie Klasse, Geschlecht, Ethnizität, Rechtsstatus oder Religion, sondern auch durch die Tatsache, dass die Wahrnehmung der grenzübergreifenden Interdependenz gestiegen ist und transnationale Interaktionen selbst zu einem Differenzkritierium geworden sind. Internationale Migration ist dabei von großer strategischer Bedeutung für das Verständnis einer transnationalen sozialen Frage, weil dadurch die Querverbindungen der fragmentierten Welten sozialer Sicherung sichtbar werden. Insbesondere stellt sich dabei die Frage, welche Mechanismen zur sozialen Sicherung über Grenzen hinweg beitragen und wie sie sowohl zur Abmilderung als auch zur Genese neuer und alter sozialer Ungleichheiten beitragen.

Stichworte: soziale Ungleichheiten, Transnationalität, Migration/Mobilität, soziale Sicherung, Europa

\section{Toward the transnational social question: social inequalities from social protection in Europe}

Summary: How do cross-border practices of social protection impact social inequalities in Europe? The contemporary, transnational social question - running along diverse lines of inequalities, such as class, gender, ethnicity, legal status, citizenship and religion and also transnationality - has implications far beyond national borders since the perception of crossborder interdependence has grown with the flow of persons, goods, capital and services. Cross-border migration is particularly relevant for understanding the transnational, social question because it links the disparate and fragmented worlds of unequal life chances and social protection. Of particular interest is the question which mechanisms contribute to social protection across borders, and this involves the reproduction of existing and the generation of new social inequalities. It is important to analyse the consequences on various scales, for example, between countries both within and across households.

Keywords: social inequalities, transnationality, migration/mobility, social protection, Europe

Autor

Prof. Thomas Faist Ph.D.

Universität Bielefeld

Fakultät für Soziologie/SFB 882

Postfach 100131

33501 Bielefeld

thomas.faist@uni-bielefeld.de 